\title{
A novel one-step quick assay for detection of SARS-COV2 antibodies across mammalian species
}

\author{
Xianjin Zhou ${ }^{\text {Corresp. } 1}$ \\ ${ }^{1}$ Department of Psychiatry, University of California San Diego, La Jolla, California, United States \\ Corresponding Author: Xianjin Zhou \\ Email address: xzhou@ucsd.edu
}

Severe acute respiratory syndrome coronavirus 2 (SARS-CoV2) has so far infected almost a hundred of millions of people and caused more than a million of death across the world. Many serological tests have been developed to track down virus infection in community via identification of antibodies against SARS-CoV2 virus. However, the tests vary in sensitivity, specificity, complexity, and speed. Here, I developed a simple, one-step, quick test to detect antibodies against SARS-CoV2 N ( $\mathrm{scN}$ ) nucleocapsid protein via direct visualization of antigen-antibody reaction. 40 serum samples of SARS-CoV2 patients were purchased from RayBiotech. 50 pre-pandemic human serum samples from San Diego Blood Bank were used as negative controls. After performing the one-step quick test of these 90 serum samples, I found that 39 samples are positive for anti-scN antibodies. All of the 39 positives are from the 40 SARS-CoV2 patients, suggesting that the one-step test is more sensitive than the lateral flow immunoassay (LFIA), the most widely used rapid antibody test. None of the 50 pre-pandemic samples is positive for anti-scN antibodies, indicating that the one-step test has an excellent specificity. The one-step test takes only $\sim 5$ minutes to detect the antibodies; and 1 milliliter of $E$. coli culture can produce reagent proteins sufficient for thousands of the tests. Since the one-step test does not need a secondary antibody, it can be used as a universal test for anti-scN antibodies across different mammalian species to track down both human infection and the animal reservoir of SARSCoV2 virus. 
1

2

3

4

5

6

7

8

9

10

11

12

13

14

15

16

17

18

19

20

21

22

23

24

25

26

27

28

29

30

31

32

33

34

35

36

37

38

39

40

41

42

\section{A Novel One-Step Quick Assay for Detection of SARS- COV2 Antibodies across Mammalian Species}

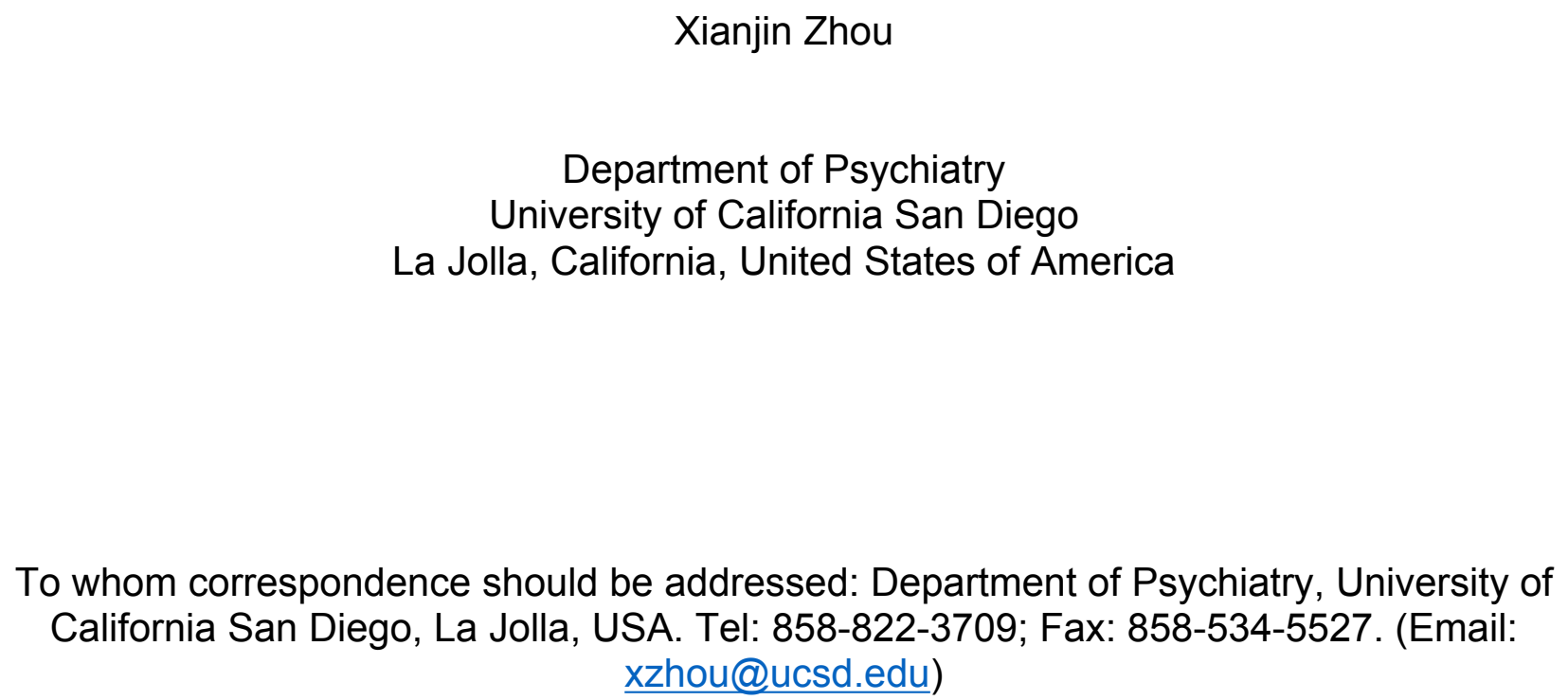

To whom correspondence should be addressed: Department of Psychiatry, University of California San Diego, La Jolla, USA. Tel: 858-822-3709; Fax: 858-534-5527. (Email: xzhou@ucsd.edu) 


\section{Abstract}

44

45 Severe acute respiratory syndrome coronavirus 2 (SARS-CoV2) has so far infected almost a hundred of millions of people and caused more than a million of death across

47 the world. Many serological tests have been developed to track down virus infection in community via identification of antibodies against SARS-CoV2 virus. However, the tests vary in sensitivity, specificity, complexity, and speed. Here, I developed a simple, one-step, quick test to detect antibodies against SARS-CoV2 N (scN) nucleocapsid protein via direct visualization of antigen-antibody reaction. 40 serum samples of SARS-CoV2 patients were purchased from RayBiotech. 50 pre-pandemic human serum samples from San Diego Blood Bank were used as negative controls. After performing the one-step quick test of these 90 serum samples, I found that 39 samples are positive for anti-scN antibodies. All of the 39 positives are from the 40 SARS-CoV2 patients, suggesting that the one-step test is more sensitive than the lateral flow immunoassay (LFIA), the most widely used rapid antibody test. None of the 50 prepandemic samples is positive for anti-scN antibodies, indicating that the one-step test has an excellent specificity. The one-step test takes only $\sim 5$ minutes to detect the antibodies; and 1 milliliter of $E$ coli culture can produce reagent proteins sufficient for thousands of the tests. Since the one-step test does not need a secondary antibody, it can be used as a universal test for anti-scN antibodies across different mammalian species to track down both human infection and the animal reservoir of SARS-CoV2 virus. 
68

69

70

71

72

73

74

75

76

77

78

79

80

81

82

83

84

85

86

87

\section{Introduction}

Antibodies, the biomarkers for a variety of human diseases, particularly infectious

diseases (1), can be detected by many laboratory immunoassays such as enzyme-

linked immunosorbent assay (ELISA), Western blot, cell-based assays,

immunohistochemistry etc. The pandemic of coronavirus disease 2019 (COVID-19)

caused by SARS-CoV2 virus creates a sense of urgency for the development of rapid

antibody tests with a high sensitivity and specificity to track down virus infection in

community and provide clinical point-of-care for patients. ELISA, lateral flow

immunoassay (LFIA), and direct chemiluminescence immunoassay (CLIA) are three

assays mostly used in serological tests of human SARS-CoV2 infection (2). These

assays vary in sensitivity, specificity, complexity, and speed. LFIA is the fastest

immunoassay that detects anti-scN antibodies in $\sim 15$ minutes (3). However, LFIA

specificity and sensitivity are often compromised by many factors, particularly the supporting nitrocellulose membrane and protein stability after drying (4). In fact, all immunoassays including ELISA and CLIA use solid support and protein coating, which makes these assays prone to non-specific binding of antibodies (5). Here, I developed a novel, one-step, quick test to detect SARS-CoV2 antibodies by direct visualization of antigen-antibody reaction in solution rather than on solid support. This avoids nonspecific background from both solid support and coated dried proteins. I selected the nucleocapsid protein as an antigen for assay development in order to compare the onestep quick test with the most widely used LFIA that detect antibodies against SARSCoV2 nucleocapsid protein. The sensitivity of the one-step quick test is superior to LFIA in the same group of SARS-CoV2 patient sera. 
92

93

94

95 96

\section{Materials and Methods}

Production of scN-GFP in E coli

Nucleocapsid protein sequence of SARS-CoV2 virus was from NCBI reference sequence database (Accession NP_828858). Gene encoding scN-GFP fusion protein with a 6 His tag was synthesized and cloned into pET-21d vector. BL21(DE3)pLysS competent $E$ coli cells were purchased from EMD (cat. 70236-3) for transformation of the plasmid. In brief, a single $E$ coli colony was inoculated in $2 \mathrm{ml}$ of LB medium containing $100 \mu \mathrm{g} / \mathrm{ml}$ carbenicillin. After overnight shaking at $37^{\circ} \mathrm{C}$, the $E$ coli culture was diluted 1:10 with LB medium containing both $100 \mu \mathrm{g} / \mathrm{ml}$ carbenicillin and $0.2 \mathrm{mM}$ IPTG. The diluted $E$ coli culture was vigorously shaked for 4 hours at $37^{\circ} \mathrm{C}$ to induce over-expression of scN-GFP proteins. After centrifugation, $E$ coli cell pellet was suspended in $1 \mathrm{X}$ PBS, $0.25 \mathrm{M} \mathrm{NaCl}, 1 \mathrm{mM}$ PMSF, and then sonicated on ice. scN-GFP proteins in the supernatant were collected after centrifugation. HisPur Ni-NTA resins (cat. 88221, ThermoFisher Scientific) were washed with 1XPBS before loaded with the scN-GFP containing supernatant. After $10 \mathrm{X}$ volume of washing with $1 \mathrm{X}$ PBS, $0.25 \mathrm{M}$ $\mathrm{NaCl}$, scN-GFP proteins were eluted with 1X PBS, $0.25 \mathrm{M} \mathrm{NaCl}, 120 \mathrm{mM}$ imidazole.

\section{One-step test}

Rabbit polyclonal antibodies against SARS-CoV2 N nucleocapsid proteins were purchased from SinoBiological (cat. 40588-T62). Protein A/G/L was purchased from Novus Biologicals (NBP2-34985) and diluted to 1ug/ul with antibody diluent solution (DAKO, S080983-2). Protein A/G/L tagged with 6 His was also over-expressed in $E$ coli and purified in my laboratory. The purified scN-GFP proteins were diluted with 1X PBS, 
$1150.25 \mathrm{M} \mathrm{NaCl}$ for use. $1 \mu \mathrm{l}$ of serum was mixed with $3 \mu \mathrm{l}$ of the diluted scN-GFP and $1 \mathrm{ul}$

116 of protein $\mathrm{A} / \mathrm{G} / \mathrm{L}(1 \mathrm{ug} / \mathrm{ul})$. After incubation for 5 minutes at room temperature, antigen-

117 antibody aggregates of the one-step test were examined for GFP green fluorescence

118 using microscope EVOS FL (ThermoFisher Scientific).

119 Human serum samples

12040 serum samples from SARS-CoV2 patients diagnosed by PCR or antigen tests were

121 purchased from RayBiotech (Supplemental data 1). Blood from 37 patients were drawn

122 33-35 days after the diagnostic test. Blood of patient $A 11, A 18$, and B7 were drawn 64,

12325 , and 18 days after the test, respectively. 50 pre-pandemic human serum samples

124 were purchased from San Diego Blood Bank (Supplemental data 2). The studies were

125 approved by Human Research Protections Program at University of California San

126 Diego.

127

\section{Results}

129

130 It is difficult to directly visualize single antigen-antibody molecule interaction for

131 detection of antibodies. I therefore propose to aggregate millions of antigen-antibody

132 molecules together to make the antigen-antibody reaction instantly visible. Protein

$133 \mathrm{~A} / \mathrm{G} / \mathrm{L}$ consists of $5 \mathrm{lg}$ G-binding region of protein $\mathrm{A}, 2 \mathrm{IgG}$-binding region of protein $\mathrm{G}$,

134 and 5 light chain-binding regions of protein L. In a mixture of antibodies and

135 fluorescence-labeled antigens, addition of protein A/G/L will instantly cross-link antigen-

136 antibody molecules into large aggregates by binding heavy and/or light chain of

137 antibodies (Figure 1A-1B). Such large aggregates will emit strong fluorescence. As a 138 proof-of-concept, SARS-CoV2 $\mathrm{N}$ nucleocapsid protein $(\mathrm{scN})$ was fused to green 
139 fluorescence protein (GFP) with a 6His tag, over-expressed in BL21(DE3) E coli cells,

140 and purified with Ni-NTA resins. Rabbit polyclonal antibodies against SARS-CoV2 N

141 nucleocapsid protein (cat. 40588-T62, SinoBiological) were diluted in human serum to a

142 concentration of $100 \mathrm{ng} / \mathrm{ul}, 10 \mathrm{ng} / \mathrm{ul}, 1 \mathrm{ng} / \mathrm{ul}$, respectively. The scN-GFP proteins were

143 diluted at $\sim 30 \mathrm{ng} / \mathrm{ul}$ and incubated with the diluted rabbit antibodies and protein A/G/L

144 (Figure 1C-1E). After 5 minute incubation at room temperature, the reactions were

145 loaded into capillary slides (cat. 76237-746, VWR) for examination. As expected,

146 antigen-antibody aggregates emit strong green fluorescence with an input of 100ng of

147 the rabbit antibodies. The limit of detection appears to be $\sim 10 \mathrm{ng}$ of the rabbit antibodies.

148 To examine non-specific background of antigen-antibody aggregates, I incubated a

149 mixture of control human serum, scN-GFP, and protein $\mathrm{A} / \mathrm{G} / \mathrm{L}$ for 5 days at $4{ }^{\circ} \mathrm{C}$ (Figure

$1501 \mathrm{~F}-1 \mathrm{G})$. No fluorescence background was observed in the antibody aggregates from

151 the control human serum, whereas strong fluorescence persists in the antigen-antibody

152 aggregates from human serum containing the rabbit antibodies against SARS-CoV2 N

153 proteins. These data suggested that the one-step quick test may have a high sensitivity

154 and specificity.

To examine whether the one-step test can detect anti-scN antibodies in patients

156

157

158

159

160

161

with SARS-CoV2 infection, I purchased 40 serum samples of patients diagnosed by PCR from RayBiotech (Supplemental data 1). Patient blood were drawn 33-35 days after the diagnostic test, except for 3 patients whose blood were drawn 64, 25, and 18 days after the test, respectively. These samples had been studied by RayBiotech for antibodies against SARS-CoV2 N proteins using LFIA rapid antibody test. Samples are deemed positive if either IgG or IgM is positive by LFIA, and 20 out of the 40 samples 
162 are positive for anti-scN antibodies. The other 20 samples are negative by LFIA. 50

163 pre-pandemic human serum samples from San Diego Blood Bank were used as

164 negative controls (Supplemental data 2). The one-step test was conducted to examine

165 anti-scN antibodies in all of the 90 serum samples. 39 positive serum samples were

166 identified, and all of them came from the 40 SARS-CoV2 patients. None of the 50 pre-

167 pandemic serum samples is positive for anti-scN antibodies (Figure 2A-2E, Controls).

168 All of the 20 patient samples deemed positive by LFIA are also identified as positive by

169 the one-step test (Figure 2F-2J, Group A). Out of the 20 patient samples deemed

170 negative by LFIA, 19 samples were identified as positive by the one-step test (Figure

$1712 \mathrm{~K}-2 \mathrm{O}$, Group B). Figure 2 shows images of some representative controls and patients.

172 Images of the rest of the controls (Supplemental Figures S1-S5) and the patients

173 (Supplemental Figures S6-S8) were provided as supplemental figures. The results are

174 summarized in Table I. As expected, antibodies against scN can be detected in the

175 patients' blood more than one month after diagnosis. How long the antibodies persist in

176 patient blood remains to be studied. The negative one by the one-step test may have a

177 very low level of anti-scN antibodies or did not develop anti-scN antibodies since the

178 patient was also negative by PCR diagnosis (supplemental data 1). In this small cohort

179 of human samples, the one-step test has $\sim 97 \%$ sensitivity and $100 \%$ specificity. In

180 conclusion, the one-step test appears to be more sensitive than LFIA.

181

182 Discussion

183

184 Current immunoassays indirectly detect antibodies. It is difficult to "see" the contribution

185 of non-specific background from solid surface and coated proteins in each reaction. 
186 The one-step test offers a new immunoassay to instantly visualize direct antigen-

187 antibody reaction in solution rather than on solid support, avoiding all non-specific

188 background except antibody cross-reactions. Reaction of antigens and antibodies in

189 solution help maintain protein structure, which may contribute to its excellent specificity.

190 Aggregating antigen-antibodies simultanouesly depletes background fluorescence,

191 which enhances its detection sensitivity. The one-step test is conducted in a small

192 reaction volume (5-6ul) to achieve a high concentration of antibodies and antigens,

193 which makes antigen-antibody reaction very fast $(<5 \mathrm{~min})$ for detection of antibodies.

194 Since the one-step test does not need a secondary antibody and protein A/G/L binds

195 antibodies from many different mammals, the exactly same test can be used to detect

196 anti-scN antibodies across mammalian species. This could be particularly helpful for

197 studies tracking down the animal reservoir of the SARS-CoV2 virus where secondary

198 antibodies against the host animals are not available for classic immunoassays. Such

199 tracking studies are important in preventing introduction and re-introduction of SARS-

200 CoV2 from animals to human population.

LFIA is the most widely used rapid antibody test currently provided by Kroger and 202 Ralph stores across the US. It detects anti-scN antibodies from past infection of SARS-

203 CoV2 virus. The LFIA sensitivity varies from different studies, in part due to using 204 different patient samples that have different levels of anti-scN antibodies. In this study, 205 the one-step test is compared with LFIA in the same group of serum samples. The one206 step test displayed a much higher sensitivity than LFIA (IgG and IgM combined 207 sensitivity) in detection of anti-scN antibodies. Although LFIA specificity was not 208 examined, the one-step test has $100 \%$ specificity in the 50 pre-pandemic human sera. 
209 Altogether, this study suggests that the one-step test be superior to LFIA in detection of

210 anti-scN antibodies. However, the one-step test examines all anti-scN antibodies (IgG,

$211 \lg \mathrm{M}, \lg \mathrm{A}, \lg \mathrm{E}$, and $\lg \mathrm{D}$ ), whereas LFIA can differentiate $\lg \mathrm{G}$ and $\lg \mathrm{M}$ anti-scN antibodies.

212 All scN-based antibody tests may be complicated by cross-reactions with SARS-CoV1

213 nucleocapsid protein that have $\sim 90 \%$ homology with the nucleocapsid protein of SARS-

214 CoV2 (6). Their spike proteins, however, are less well conserved with $79.6 \%$ identity.

215 Specific antigenic epitopes may be selected from the spike protein for the development

216 of assays to differentiate their infections. It is unlikely that antibodies aganist

217 nucleocapsid protein of SARS-CoV2 may cross-react with the nucleocapsid proteins of

218 other flu-causing coronavirus due to their limited homology. Consistently, the one-step

219 test did not detect any positive signals from the 50 pre-pandemic sera. More studies

220 are needed to investigate cross-reactions between the anti-scN antibodies and antigens

221 of other viruses.

222 A potential complication of the one-step test could arise from antibodies

223 recognizing the GFP part of the fusion protein rather than the antigen part. However, I

224 did not find any human sera binding GFP after screening 200 human serum samples.

225 Such false positives, if occur, can be readily ruled out by incubating the sera with GFP

226 proteins. As an alternative approach to avoid potential cross-reaction of serum

227 antibodies with GFP proteins, a small fluorescence dye such as fluorescein

228 isothiocyanate (FITC) or Alexa Fluor rather than GFP could be conjugated to scN

229 antigen for the one-step test. Different sera may have different levels of

230 autofluorescence metabolites and/or proteins. The intensity of the green

231 autofluorescence is, however, far less than the fluorescence intensity of the antigen- 
232 GFP proteins added into the assay. I only examine the increase of green fluorescence

233 of large aggregated antibody-antigen-GFP complexes above the green fluorescence

234 background that is mainly determined by free antigen-GFP proteins. Blood

235 autofluorescence from soluble metabolites or proteins may slightly contribute to

236 variations of background between individual samples. Since these soluble

237 autofluorescence metabolites or proteins are not incorporated into the aggregates, they

238 have little effect on the one-step test. I do not encounter a problem from blood

239 autofluorescence in the assay. However, GFP tag can be replaced with a different

240 fluorescence protein to avoid autofluorescence if needed.

An advantage of the one-step test is that both scN-GFP and protein $\mathrm{A} / \mathrm{G} / \mathrm{L}$ can be

242 abundantly produced in $E$ coli. $1 \mathrm{ml}$ of $E$ coli culture can generate reagent proteins

243 enough for thousands of the one-step tests. Purified scN-GFP and protein A/G/L are

244 stable for months in $4{ }^{\circ} \mathrm{C}$ refrigerator. Therefore, the one-step test could be particularly

245 attractive for developing countries to produce sufficient diagnostic tests to track down

246 SARS-CoV2 infection during the pandemic. In the future, development of smartphone

247 fluorescence microscope (7) will enable the one-step test to be conducted by

248 individivals at home.

249 This study demonstrated the utilization of the one-step test in detection of anti$250 \mathrm{SCN}$ antibodies. Different antigens can however be labeled with fluorescence or other

251 dyes for detection of their antibodies after aggregation. If SARS-CoV2 RBD is fused

252 with GFP, the test would detect neutralizing antibodies against SARS-CoV2 virus. This

253 could be particularly helpful for persons to monitor the levels of circulating SARS-CoV2

254 neutralizing antibodies after vaccination. More broadly, this strategy may be used to 
255 develop various one-step tests to detect different antibodies involved in a variety of 256 human and animal diseases.

257

258

259

260

261

262

263

264

265

266

267

268

269

270

271

272

273

274

275

276

277

278

279

280

281

282

283

284

285

286

287

288

289

290

291

292

293

294

295

296

297 
298

299

300

301

302

303

304

305

306

307

308

309

310

311

312

313

314

315

316

317

318

319

320

321

322

323

324

325

326

327

328

329

330

331

332

333

\section{References}

1. Peruski AH, Peruski LF, Jr. (2003): Immunological methods for detection and identification of infectious disease and biological warfare agents. Clin Diagn Lab Immunol. 10:506-513.

2. Espejo AP, Akgun Y, Al Mana AF, Tjendra Y, Millan NC, Gomez-Fernandez C, Cray C (2020): Review of Current Advances in Serologic Testing for COVID-19. American journal of clinical pathology. 154:293-304.

3. Li Z, Yi Y, Luo X, Xiong N, Liu Y, Li S, Sun R, Wang Y, Hu B, Chen W, Zhang Y, Wang J, Huang B, Lin Y, Yang J, Cai W, Wang X, Cheng J, Chen Z, Sun K, Pan W, Zhan Z, Chen L, Ye F (2020): Development and clinical application of a rapid IgM-lgG combined antibody test for SARS-CoV-2 infection diagnosis. J Med Virol. 92:1518-1524.

4. Pavlova IP, Nair SS, Kyprianou N, Tewari AK (2020): The Rapid Coronavirus Antibody Test: Can We Improve Accuracy? Front Med (Lausanne). 7:569.

5. Terato K, Do C, Chang J, Waritani T (2016): Preventing further misuse of the ELISA technique and misinterpretation of serological antibody assay data. Vaccine. 34:4643-4644.

6. Dutta NK, Mazumdar K, Gordy JT (2020): The Nucleocapsid Protein of SARSCoV-2: a Target for Vaccine Development. J Virol. 94.

7. Dai B, Jiao Z, Zheng L, Bachman H, Fu Y, Wan X, Zhang Y, Huang Y, Han X, Zhao C, Huang TJ, Zhuang S, Zhang D (2019): Colour compound lenses for a portable fluorescence microscope. Light Sci Appl. 8:75. 
334 Figure Legends

335

336

337

338

339

340

341

342

343

344

345

346

347

348

349

350

351

352

353

354

355

356

357

Figure 1. Development of the one-step test for detection of antibodies against

SARS-CoV2 N nucleocapsid protein. (A) A strategy of direct visualization of antigenantibody reaction via aggregation by protein $\mathrm{A} / \mathrm{G} / \mathrm{L}$. Antigen is labeled with GFP. Protein A/G/L cross-links Ig Fc and/or light chain of all antibodies (IgG, $\lg M, \lg A, \lg E$, and $\operatorname{lgD}$ ) that bind antigen-GFP to form high fluorescence aggregates. Aggregating fluorescent antigen-antibody complexes simultaneously depletes background fluorescence to achieve a high sensitivity. (B) One-Step assay. Rabbit polyclonal antibodies against SARS-CoV2 N nucleocapsid proteins were diluted in human serum. Different amount of the rabbit antibodies (100ng (C), 10ng (D), 1ng (E)) was incubated with the scN-GFP fusion proteins for 5 minutes to examine the detection limit of the onestep test. Blackwhite images show antigen-antibody aggregates, and green fluorescence of the aggregates is shown side by side. Bar: 100um. Non-specific background of the antigen-antibody aggregates was examined after 5 day incubation of reactions at $4^{\circ} \mathrm{C}$. (F) A pre-pandemic human serum was used as a negative control. (G) A positive control was human serum containing 100ng of the rabbit antibodies against SARS-CoV2 N nucleocapsid protein.

Figure 2. Sensitivity and specificity of the one-step test in patient serum samples. (A) The positive control was human serum containing 100ng of the rabbit antibodies against SARS-CoV2 N nucleocapsid protein. The negative controls are 4 representative samples (SDBB1-4 (B-E)) from the 50 pre-pandemic human serum samples from San Diego Blood Bank (SDBB). Patient Group A has 20 SARS-CoV2 
358 patient sera deemed positive (either IgG or IgM positive) by LFIA conducted by

359 RayBiotech. All of the 20 patient samples are positive for anti-scN antibodies by the

360 one-step test. (F-J) 5 representatives of the 20 positive samples are shown. Patient

361 Group B has 20 SARS-CoV2 patient serum samples deemed negative (both IgG and

362 IgM are negative) by LFIA conducted by RayBiotech. 19 out of the 20 LFIA negative

363 samples are positive for anti-scN antibodies by the one-step test. (K-O) 5

364 representatives of the 19 positive samples are shown. 


\section{Figure 1}

Development of the one-step test for detection of antibodies against SARS-CoV2 N nucleocapsid protein

(A) A strategy of direct visualization of antigen-antibody reaction via aggregation by protein A/G/L. Antigen is labeled with GFP. Protein A/G/L cross-links Ig FC and/or light chain of all antibodies (IgG, $\lg M, \lg A, \lg E$, and $\lg D)$ that bind antigen-GFP to form high fluorescence aggregates. Aggregating fluorescent antigen-antibody complexes simultaneously depletes background fluorescence to achieve a high sensitivity. (B) One-Step assay. Rabbit polyclonal antibodies against SARS-CoV2 N nucleocapsid proteins were diluted in human serum.

Different amount of the rabbit antibodies (100ng (C), 10ng (D), 1ng (E)) was incubated with the scN-GFP fusion proteins for 5 minutes to examine the detection limit of the one-step test. Blackwhite images show antigen-antibody aggregates, and green fluorescence of the aggregates is shown side by side. Bar: 100um. Non-specific background of the antigenantibody aggregates was examined after 5 day incubation of reactions at $4{ }^{\circ} \mathrm{C}$. (F) A prepandemic human serum was used as a negative control. (G) A positive control was human serum containing $100 \mathrm{ng}$ of the rabbit antibodies against SARS-CoV2 $\mathrm{N}$ nucleocapsid protein. 

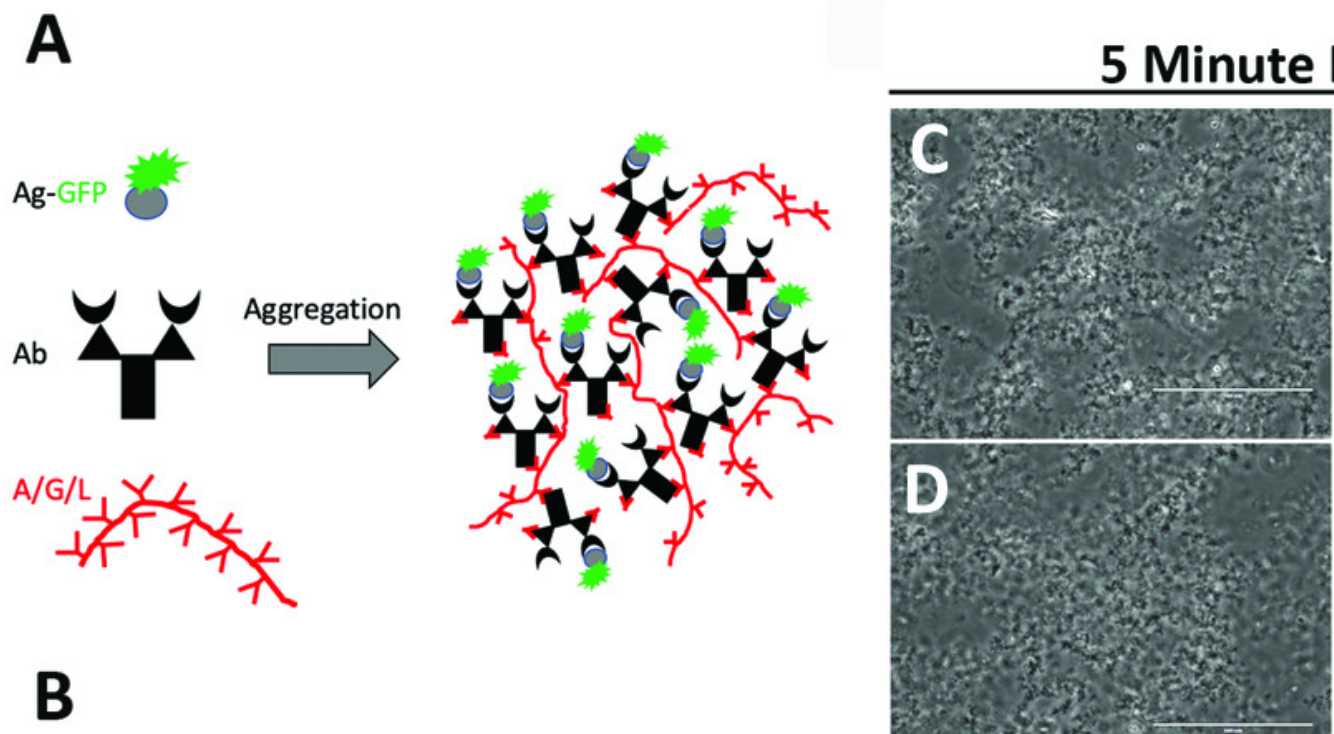

\section{ncubation}


5 Day Incubation

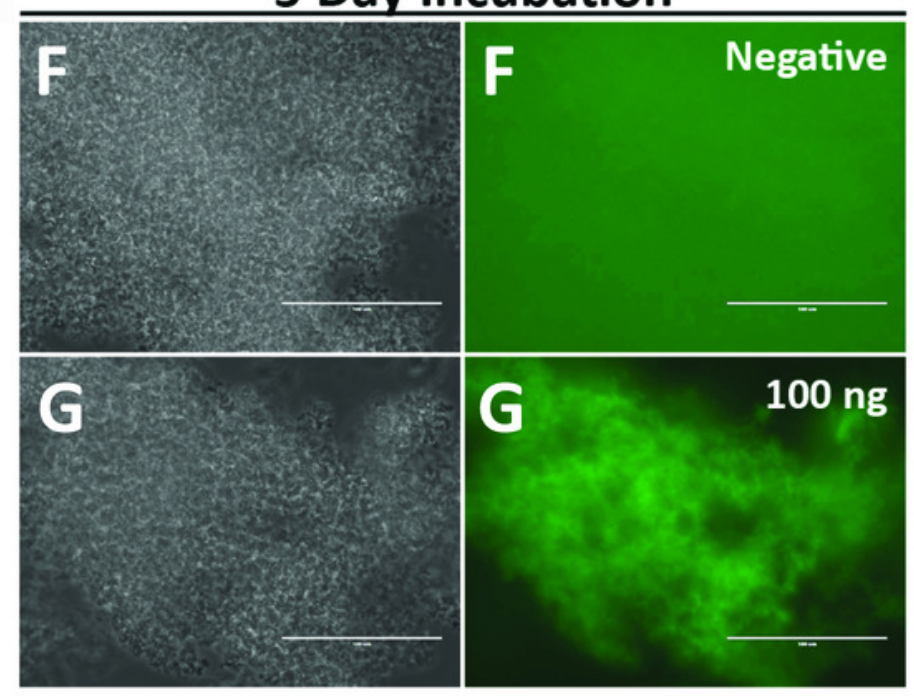




\section{Figure 2}

Sensitivity and specificity of the one-step test in patient serum samples.

(A) The positive control was human serum containing 100ng of the rabbit antibodies against SARS-CoV2 $\mathrm{N}$ nucleocapsid protein. The negative controls are 4 representative samples (SDBB1-4 (B-E)) from the 50 pre-pandemic human serum samples from San Diego Blood Bank (SDBB). Patient Group A has 20 SARS-CoV2 patient sera deemed positive (either IgG or IgM positive) by LFIA conducted by RayBiotech. All of the 20 patient samples are positive for anti-scN antibodies by the one-step test. (F-J) 5 representatives of the 20 positive samples are shown. Patient Group B has 20 SARS-CoV2 patient serum samples deemed negative (both IgG and IgM are negative) by LFIA conducted by RayBiotech. 19 out of the 20 LFIA negative samples are positive for anti-scN antibodies by the one-step test. (K-O) 5 representatives of the 19 positive samples are shown. 


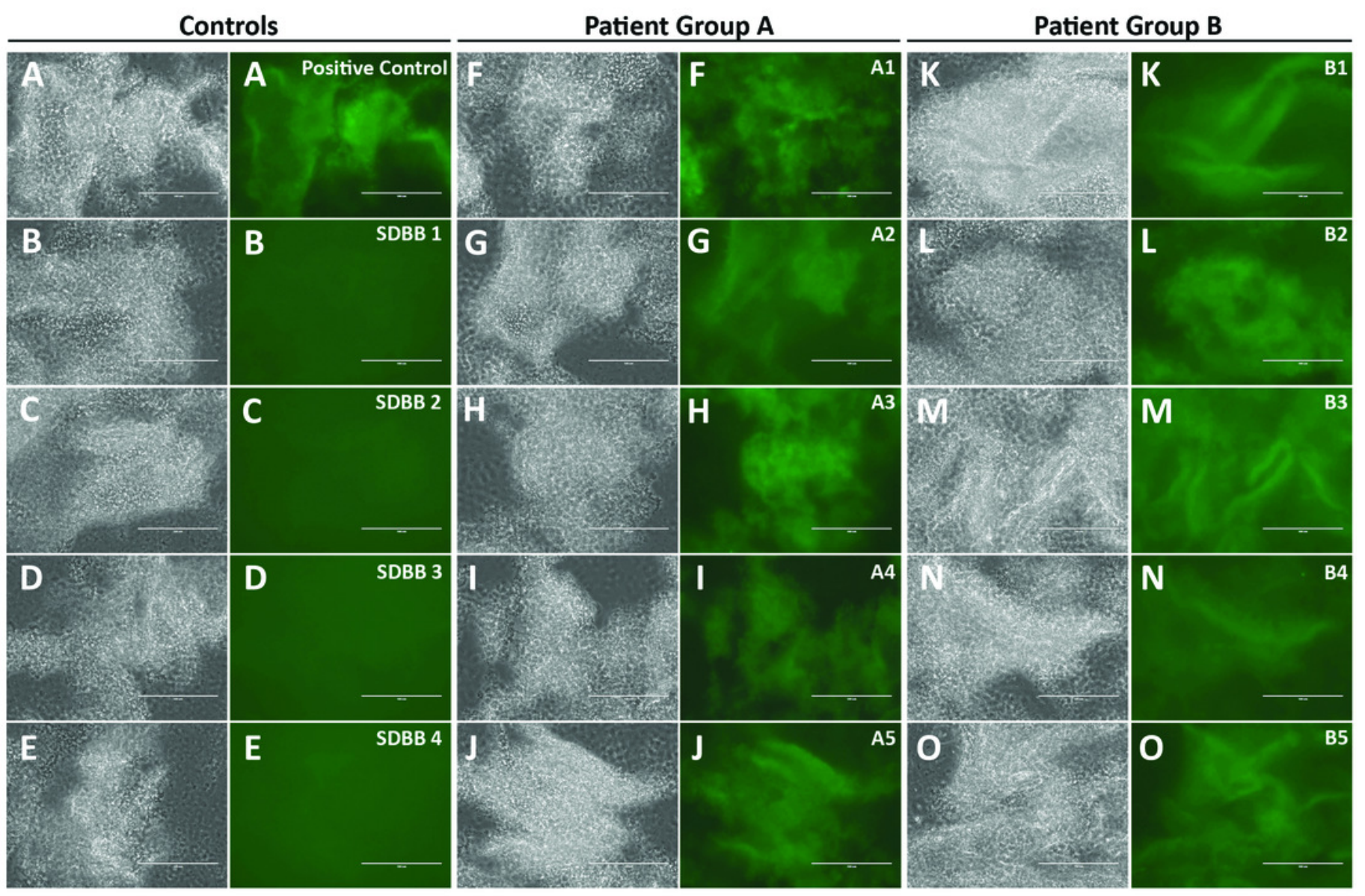




\section{Table $\mathbf{1}$ (on next page)}

Comparison between LFIA Rapid Antibody Test and the One-Step Test

Comparison between LFIA Rapid Antibody Test and the One-Step Test 
Table I. Comparison between LFIA Rapid Antibody Test and the One-Step Test

\begin{tabular}{|c|c|c|c|}
\hline & \multicolumn{2}{|c|}{ RayBiotech Patients } & SDBB Pre-Pandemic Controls \\
\cline { 2 - 3 } & Group A ( $\mathrm{n}=20)$ & Group B ( $\mathrm{n}=20)$ & $52.5(17.9)$ \\
\hline Age (SD) & $64.3(16.1)$ & $64.1(17)$ & N/A \\
Positives by LFIA & 20 & 0 & 0 \\
Positives by One-Step & 20 & 19 & \\
\hline
\end{tabular}

1 